\title{
Chronic Inflammatory Diseases and Endothelial Dysfunction
}

\author{
Xavier Castellon*, Vera Bogdanova \\ Privat Hospital, Athis Mons, 91200 Athis-Mons, Paris, France
}

[Received November 21, 2014; Revised August 1, 2015; Accepted August 3, 2015]

\begin{abstract}
Chronic inflammatory diseases are associated with increases in cardiovascular diseases (CVD) and subclinical atherosclerosis as well as early-stage endothelial dysfunction screening using the FMD method (Flow Mediated Dilation). This phenomenon, referred to as accelerated pathological remodeling of arterial wall, could be attributed to traditional risk factors associated with atherosclerosis. Several new non-invasive techniques have been used to study arterial wall's structural and functional alterations. These techniques (based of Radio Frequency, RF) allow for an assessment of artery age through calculations of intima-media thickness (RF- QIMT), pulse wave rate (RF- QAS) and endothelial dysfunction degree (FMD). The inflammatory and autoimmune diseases should now be considered as new cardiovascular risk factors, result of the major consequences of oxidative stress and RAS (Renin Angiotensin System) imbalance associated with the deleterious effect of known risk factors that lead to the alteration of the arterial wall. Inflammation plays a key role in all stages of the formation of vascular lesions maintained and exacerbated by the risk factors. The consequence of chronic inflammation is endothelial dysfunction that sets in and we can define it as an integrated marker of the damage to arterial walls by classic risk factors. The atherosclerosis, which develops among these patients, is the main cause for cardiovascular morbi-mortality and uncontrolled chronic biological inflammation, which quickly favors endothelial dysfunction. These inflammatory and autoimmune diseases should now be considered as new cardiovascular risk factors.
\end{abstract}

Key words: endothelial dysfunction, atherosclerosis, inflammatory diseases, non-invasive artery age testing methods, FMD

Aging Chronic inflammatory diseases are associated with increases in cardiovascular diseases (CVD), subclinical atherosclerosis prevalence and outcomes as well as earlystage endothelial dysfunction screening using the FMD method (Flow Mediated Dilation). Such diseases may occur before the appearance of a clinical illness and therefore act as an early identification objective $[1,2]$.

This phenomenon, referred to as accelerated pathological remodeling, could be attributed to traditional risk factors associated with atherosclerosis. However, it might also be the result of other autoimmune and inflammatory mechanisms that are aggravated with chronic inflammatory diseases and more so with certain recently studied diseases such as chronic inflammatory arthritis and connective diseases. This remodeling of the arterial wall is accelerated for the above-mentioned diseases as the associated inflammatory disorders and autoimmune mechanisms can work in synergy with traditional risk factors, which makes them particularly harmful and can therefore contribute to endothelial dysfunction, considered to be the first step towards atherogenesis. These inflammatory and autoimmune diseases should now be considered as new cardiovascular risk factors. These non-traditional risk factors are said to contribute to atherogenesis via several mechanisms: autoantibodies against phospholipids: Anti Phospholipid 
Syndrome (APS); against cells: ds-ADN endothelial dsDNA (anti-double-stranded DNA antibodies (antidsDNA) purified from patients with active systemic lupus erythematosus (SLE) or neutrophil polynuclear anti cytoplasmic antibodies (NP), systemic inflammation, acute lipid oxidation, thermal shock proteins (HSP); antibodies against HSPs, association of anti-oxld1/2 Gp1 OxLDL/beta2GPI-anti-oxLDL/beta2GPI complex and atherosclerosis in SLE patients, immune complex and accelerated atherosclerosis in patients with primary APS oxLDL/B2GP1 and anti-oxLDL/B2GP1 $[1,3,4]$.

We currently use several new non-invasive techniques to study arterial walls' structural and functional alterations.These non-invasive techniques allow for an assessment of artery age via calculations of intima-media thickness (RF- QIMT), pulse wave rate (RF- QAS) and endothelial dysfunction degree by FMD. These are currently the only reliable markers, evidencing subclinical arterial damage caused by risk factors and chronic inflammatory diseases. Such screening is justified with these patients in order to assess the degree of accelerated arterial wall aging associated with screening of the atheromatosis and its progression, and to also assess the state of the endothelial function and therefore the actual cardiovascular risk thereby allowing for a better care management strategy and a decrease in the occurrence of cardiovascular events [5-7].

\section{Vessel structure alterations as part of artery aging due to old age}

From a histological standpoint, the alterations pertain to all artery linings. Intima media thickness considerably increases with age, which is a result of extracellular molecular and smooth muscular cell cluttering. In the intima media (level of arterial wall), the endothelial cells feature alterations with a less unclear alignment, flatter cells or, on the contrary, hypertrophic or irregular cells. In the media, elastin destruction and the increase in collagen contents inverts the elastin/collagen ratio, followed by collagen stiffening and a decrease in elasticity. These structural arterial wall alterations are evidenced by the occurrence of vasomotricity stiffening and alteration as years go by. Such vascular aging is accelerated by the presence of non-treated or noncontrolled risk factors and chronic inflammatory diseases, which favor atheromatous progression and ultimately result in severe endothelial dysfunction. These changes quickly increase the arteries' sensitivity to hypertension, atherosclerosis, medial degeneration and the initiation of another set of artery complications (i.e. myocardial infarctions, strokes, aneurisms)[8-12].

\section{Chronic inflammation}

Oxidative stress is the basic etiology of the alteration of the arterial wall; it is an imbalance between of antioxidants and free radicals or, between the enzymatic systems of oxygen and free radicals. This imbalance causes side effects by decreasing the bio- availability of Nitric Oxide (NO), Arginine and Adenosine TriPhosphate (ATP), a destructive cascade identified at the arterial wall, followed by the initiation of chronic inflammation, and dysfunction of the endothelium. The dysfunction of protective mechanisms against atherosclerosis leads to vascular dysfunction and the progressive onset from subclinical lesions to clinical pathological manifestations. Causes that promote oxidative stress are: hypoxia, respiratory diseases favored by repeated pulmonary infections, smoking, frequent exposure to the sun, alcohol and air pollution [13-16].

The key role of the Renin Angiotensin System (RAS) in vascular disease: participation and remodeling of the arterial wall. Stimulation of the Angiotensin II AT1 receptor level increases the production of cell adhesion, which allows monocytes to infiltrate the endothelium, and transform into macrophages, and become foam cells. These cells secrete cytokines and inflammation markers (Interleukins, CRP) following the assault. The inflammatory response then begins and alters the arterial wall. The first manifestation of this infrastructural disease remodeling is a disruption of elastic blades of the intima media, with migration of smooth muscle cells to the subendothelial space that transforms into fibrocytary or myofibrocytary cells [17-20].

Chronic inflammation: Chronic inflammation is a result of the major consequences of oxidative stress and RAS imbalance associated with the deleterious effect of known risk factors that lead to the alteration of the arterial wall. Inflammation plays a key role in all stages of the formation of vascular lesions maintained and exacerbated by risk factors. These result in changes in the arterial wall such as geometric vascular remodeling (the increase in intima-media thickness) and functional remodeling. And with time leads to endothelial dysfunction, its homeostatic properties and the loss of the key role in protection against atherosclerosis.

The consequence of chronic inflammation is endothelial dysfunction that sets in and we can define it as an integrated marker of the damage to arterial walls by classic risk factors. We can also evaluate their ability to repair and thus become an important factor in the development and progression of the atherosclerotic process and its consequences. However, this is also the 
case in the prognosis of patients with risk factors $[16,21$ 24].

Pathogenesis of atherosclerosis and endothelial dysfunction as part of chronic inflammatory diseases

Among recently studied chronic inflammatory diseases, which are involved in atherosclerosis and endothelial dysfunction pathogenesis, one finds inflammatory arthritis, autoimmune and connective diseases such as erythematous lupus and the anti-phospholipid syndrome, currently considered to be potential cardiovascular risk factors. These accelerate the vessel aging process and increase the general cardiovascular risk, just as metabolic syndrome, obesity, diabetes, chronic renal insufficiency and sleep apnea syndrome (SAS) do [25, 26].

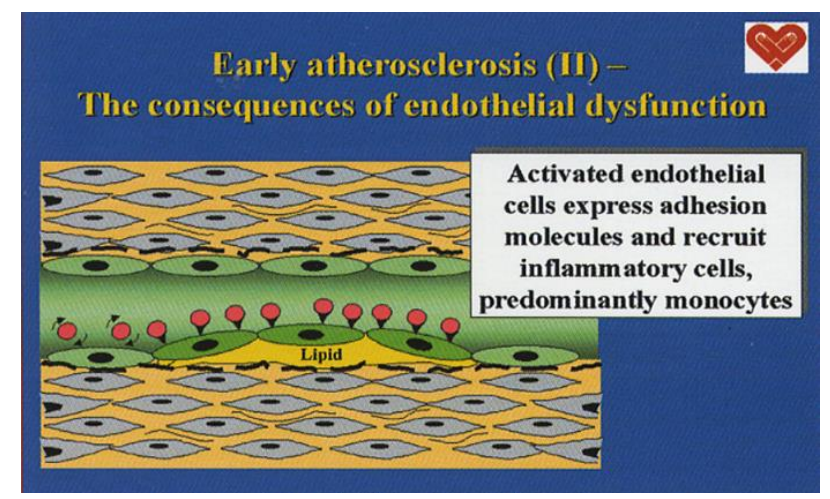

Figure 1. The genesis of the inflammatory plaque and endothelial dysfunction. Penetration and accumulation of lipoproteins in the intima LDL = by passive phenomenon Modification of LDL oxidized LDL.

\section{Atherosclerosis pathogenesis}

These traditional risk factors are said to contribute to atherogenesis via several complex mechanisms: autoantibodies against phospholipids and against cells, dsDNA, endothelial or ANCA, systemic inflammation, acute lipid oxidation, thermal shock proteins (HSP) and antibodies against HSPs, oxLDL/B2GP1 and antioxLDL/B2GP1. The atherosclerosis, which develops among these patients with cardiovascular risk factors and inflammatory syndrome, is the main cause for cardiovascular morbi-mortality and uncontrolled chronic biological inflammation, which quickly favors endothelial dysfunction $[27,28]$.

\section{Endothelial dysfunction pathogenesis}

We now describe the mechanism of endothelial dysfunction for these diseases, firstly covering chronic rheumatic and connective diseases, which currently cause the most cardiovascular damage throughout the world as a result of their frequency and aggressiveness (Figure 1).

\section{Chronic inflammatory and connective tissue diseases}

Rheumatoid arthritis and connective tissue diseases such as erythematous lupus and anti-phospholipid syndrome are autoimmune diseases, which are involved in atherosclerosis and endothelial dysfunction pathogenesis. These autoimmune diseases are associated with an increase in cardiovascular diseases due to the accelerated atherosclerosis they cause and their frequently observed associations with traditional cardiovascular risk factors and specific chronic inflammatory pathology factors. They are currently considered to be non-traditional risk factors and are associated with an increase in coronary and cerebral-vascular mortality. We will mainly discuss rheumatoid arthritis, a very frequent autoimmune disease that is known for favoring important and accelerated atherosclerosis and an aggravated inflammatory reaction in the articulations and arterial walls, with an acute cardiovascular risk [26, 28, 29] (Figure 2).

\section{Endothelial dysfunction in rheumatoid polyarthritis}

The pathophysiological mechanisms of endothelial dysfunction in rheumatoid arthritis are not well known. Arginase regulates the vascular NO level, as this enzyme competes with NO synthase (NOS) for a common substrate, L-Arginine. The alteration of arginine transportation in the endothelial cell could be one of the alteration mechanisms of way of NO as part of the aging process. There is currently knowledge of the existence of Arginase activity and expression anomalies in vessels, as well as of this enzyme's role in endothelial dysfunction. This anomaly has allowed us to identify a new endothelial dysfunction mechanism associated with chronic arthritis, suggesting that Arginase could constitute a new therapeutic prevention target [30-33].

\section{Hypoxia (Sleep Apnea Syndrome SAS)}

The SAS (Sleep Apnea Syndrome) as two immediate pathophysiological effects, which can be associated with endothelial dysfunction and arterial lesions. As part of hypopnea and apneas, intermittent drops in arterial oxygen saturation, referred to as intermittent hypoxia (IH) occur, and are considered to be responsible for most harmful impacts on arterial walls and the heart. This IH 
causes an acute formation of oxygen radicals and consequently oxidative stress, and an intermittent systemic and cellular inflammation increase, as well as the consequences of these factors on the endothelial function at the expense of SAS patients' cardiovascular health. The persistence of combined SAS and inflammation is involved in the development of arterial stiffness and endothelial dysfunction [34-36].

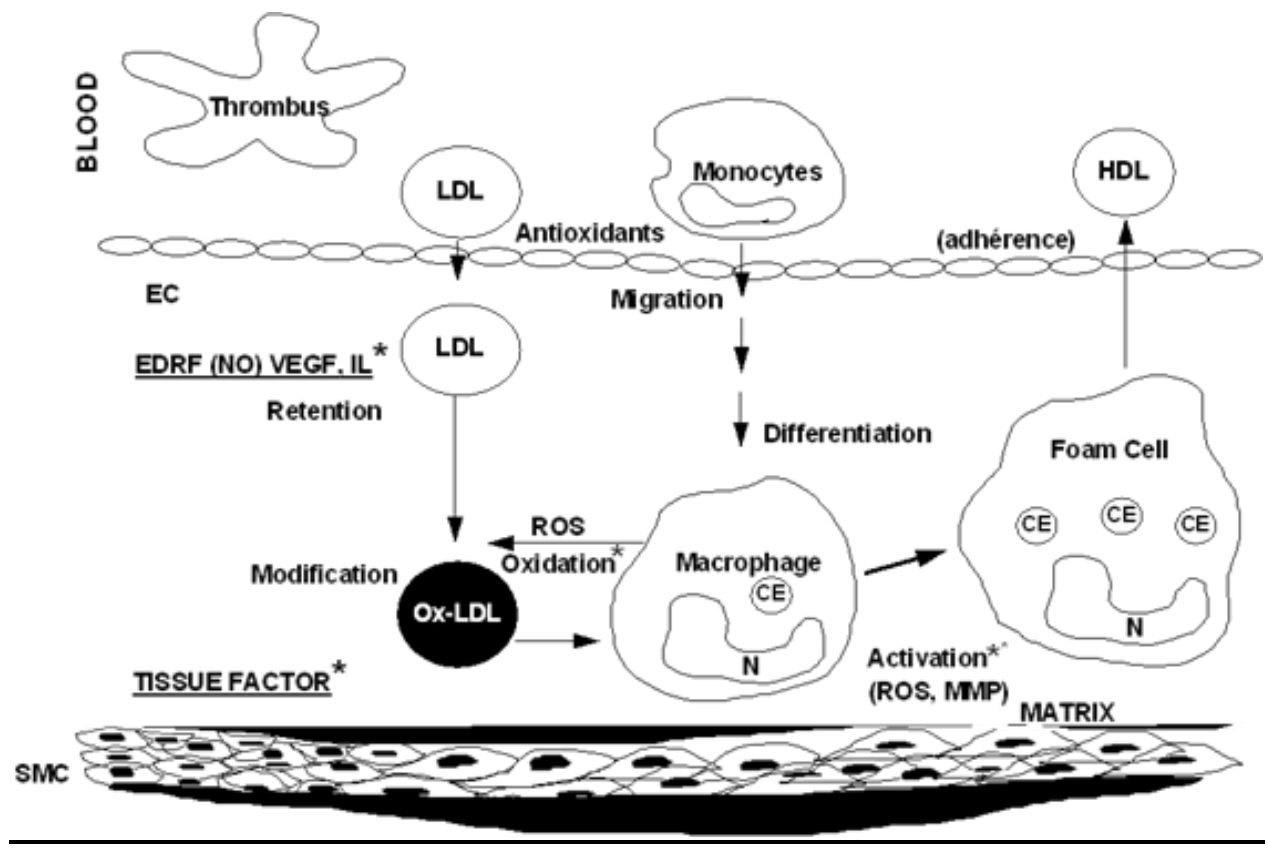

Fgiure 2. Chronic inflammatory diseases and endothelial dysfunction.

\section{Diabetes}

The loss of the endothelium's mandatory role in vasomotor modulation is a primary target for diabetes. With all the endothelium's functions being disrupted, it favors pathological vascular remodeling and plaque development. The atherosclerosis, which develops among these patients, is the main cause for cardiovascular morbimortality and uncontrolled chronic biological inflammation, which quickly favors endothelial dysfunction $[37,38]$.

\section{Endothelial dysfunction pathogenesis}

Hyperglycemia, being a direct stimulus of oxidative stress, causes NO inactivation through an excess production of free radicals, advanced glycation and a decrease in free radical removal. The excess free radicals contribute to the harmful effects on the endothelium incurred by high glucose concentrations. Disruption of the L-arginine-NO metabolic pathway, an essential pathway for vasomotricity homeostasis, appears to be essential for the development of vasculopathy and atherosclerosis, and leads to a decrease in vasodilatation, anti-platelet and antithrombotic activities as well as structural alterations [39, 40].

\section{Obesity and Metabolic Syndrome:}

Obesity and metabolic syndrome are factors considered to accelerate aging of the arteries and the early development of atherosclerosis. The uncontrolled chronic biological inflammation, which quickly favors endothelial dysfunction, and develops among the atherosclerosis, and is the main cause for cardiovascular morbi- mortality. 


\section{Endothelial dysfunction pathogenesis}

The accumulation of visceral and abdominal fat tissue, its secretion products and the decrease in adiponectin plasmatic concentration, which is the protein secreted by adipocytes and macrophages, are responsible for unfavorable effects on the whole vascular wall. New research is currently focusing on the mechanisms and causes of this endothelial dysfunction, which is the earliest vascular anomaly for these patients.

In metabolic syndrome, all components are endothelium aggression factors and mutually take part in altering endothelial functions with a decrease in NO (Nitric oxide) bioavailability. The central elements seem to be an increase in oxidative stress and a decrease in local NO bioavailability. In obesity, preadipocytes and macrophages present in fat tissue of the obese person have important adipokine production and secretion functions as well as pro-inflammatory cytokines (IL-1b, IL-6, IL-8, MCP-1 and TNF-alpha) released by the preadipocytes. Three elements can explain the link between obesity and chronic inflammation, namely: the inflammation could be a response to oxidative stress, local hypoxia and/or activation of the endoplasmic reticulum. These three hypotheses are may be associated with endoplasmic reticulum stress and the generation of oxygen free radicals potentially caused by hypoxia, the latter therefore being the main cause of fat tissue inflammation [41, 42]

\section{$\underline{\text { Chronic Renal Insufficiency (CRI) }}$}

The aging process often associated with traditional risk factors, entails an accelerated remodeling of the arterial wall. This remodeling's progress during the CRI, gives credit to the assumption that renal dysfunction is responsible for this vascular process and the vessels respond with structural ("remodeling") and functional adaptations (a decrease in vessel distensibility), as well as vasomotor tone anomalies and plaque development. Cardiovascular diseases are the first cause of morbidity and mortality for patients suffering from renal insufficiency. This can be linked to the numerous cardiovascular complications occurring before the chronic renal insufficiency (CRI) stage and/or to the primary cause for kidney ailments (such as diabetes, vascular nephropathies and hemodynamic anomalies associated with renal insufficiency itself.

\section{Endothelial dysfunction and CRI}

The causes of endothelium alteration are unclear, but it seems to be linked to chronic endothelial cell activation and their subsequent dysfunction. This is objectivized on a biological level by an increase in the blood concentrations of the many humoral factors that are synthesized and regulated by the endothelium, such as: endothelin, von Willebrand factor, plasminogen activator type 1, plasminogen's inhibitor and activator, etc., and the activation of receptors which are sensitive to neuro humoral agents. Histological research has shown the existence of sub epidermal capillary micro-angiopathy in patients suffering from renal insufficiency, which is evidenced among other things by the presence of activation and an apoptosis of endothelial cells. This initial endothelial lesion leads to vasoconstriction followed by diffuse platelet aggregation, and the occlusion of vascular light by fibrinous thrombins [43, 44].

\section{Assessment review - Atheromatosis and endothelial function in patients suffering from a chronic inflammatory disease}

Any assessment should start by thorough questioning followed by a clinical examination aiming to find clinical atheromatosis markers, as well as paraclinical assessment tests for atheromatosis and its complications and an assessment of arterial remodeling [45-47].

\section{Questioning}

Personal history (search for risk factors): 1 - High blood pressure, 2 - Tobacco, 3 - Dyslipidemia, 4 - Malnutrition, 5 - Diabetes, 6 - Obesity, 7 - BMI, 8 Sedentary lifestyle. Risk factors associated with chronic inflammatory diseases increase the probability of an occurrence of a cardiovascular (CV) event.

\section{Clinical examination}

Comprehensive cardiovascular clinical examination to look for clinical markers and cardiovascular or renal complications pertaining to atheromatosis plaque.

\section{Clinical markers}

Peripheral arterial (systolic) pressure, central pressure (CP), central and peripheral pulse pressure, average TA, arm-ankle systolic index, waist measurement, body mass index (BMI), prehypertension, LVH (left ventricular hypertrophy), basic clinical examination for patients suffering from hypertension and diabetes.

\section{Non-invasive exploration of the atheromatosis plaque and its complications}

Non-invasive CV (cardiovascular) imaging techniques: arterial Doppler Ultrasonography of the neck and lower 
limbs, stress test, coro-scanner and calcium scoring, TTE (trans thoracic echocardiography), renal ultrasonography and Doppler ultrasonography of renal arteries and the research for silent ischemia myocardial ischemia (by methods like a stress ultrasonography, myocardial perfusion, stress MRI especially for diabetic patients) [48, 49].

\section{Structural and functional exploration of the arterial wall (arterial age)}

\section{Screening TECHNIQUES for pathological remodeling of the artery wall in the coronary and carotid}

Integrating markers:

- QIMT (carotid artery) studies the geometrical alterations of the arterial wall (intima media thickness).

- QAS studies the artery's functional alterations (pulse wave speed and stiffness coefficient).

- PVI ultrasonography (carotid stress).

- MRI carotid velocimetry studies the arterial wall constraints.

- FMD method (at the humeral artery) classically studies the vasodilator function of the endothelium, and is used to assess the endothelial dysfunction level.

If this exploration of the endothelial function does not yield conclusive results, it should be further supported by the serum markers analysis of the endothelial function [50-55].

\section{Biological markers of oxidative and biochemical stress Biological markers}

General assessment to be made, pertaining to biological inflammation and an assessment adapted to each patient with specific biological markers according to the chronic illness to study. Specific biological markers for atheromatosis are currently still in their experimental stage. Other non-specific biological markers required to study the endothelial function include: micro albuminuria, Homocysteinemia and the von Willbrand factor.

\section{Oxidative stress markers:}

TBARS (nmol/gHb), plasmatic, TBARS $(\mu \mathrm{mol} / \mathrm{L})$, Ox LDL (UI/L), protein carbonyls ( $\mu \mathrm{mol} / \mathrm{gprot})$, vitamin A ( $\mu \mathrm{mol} / \mathrm{l}), \alpha$-tocopherol $(\mathrm{mg} / \mathrm{L}), \mathrm{Cu} / \mathrm{Zn}$ SOD $(\mathrm{UI} / \mathrm{g} \mathrm{Hb})$, carotene, $\mathrm{Ce}, \mathrm{Se}$, plasmatic vitamins [5, 56-58].

\section{Biochemical assessment}

Serum markers of endothelial function: NO, asymmetrical dimethyl-arginine (acute inflammation expression), NO synthase, and cGMP $[59,60]$.

\section{Therapeutic strategies}

All treatments to be carried out for these patients should on one hand control the biological inflammation of the basic disease and the risk factors, and on the other hand slow down atheromatosis and restore the endothelium. This strategy will in the future remain the best approach to reduce cardiovascular events for the chronic inflammation patient population $[59,61]$.

\section{Conclusion}

Chronic inflammatory diseases are associated with an increase in cardiovascular diseases (CVD), subclinical atherosclerosis prevalence and outcomes as well as earlystage endothelial dysfunction screening using the FMV method, which can occur before the appearance of a chronic disease and therefore be considered as an early identification objective. This phenomenon, referred to as accelerated pathological remodeling, can be attributed to traditional risk factors associated with atherosclerosis, but could also be the result of other autoimmune and inflammatory mechanisms that are aggravated with chronic inflammatory diseases and more so with certain recently studied diseases such as chronic inflammatory arthritis and connective diseases. This remodeling of the arterial wall is accelerated for the above-mentioned diseases as the associated inflammatory disorders and autoimmune mechanisms can work in synergy with traditional risk factors, which makes them particularly harmful and can therefore contribute to endothelial dysfunction, considered to be the first step towards atherogenesis. The he biological inflammation and the endothelial dysfunction is oxidative stress, a common mechanism for all chronic inflammatory diseases and vasculopathy appearance. Since 2007, non-invasive imaging has been used in endothelial dysfunction diagnoses, which allow a detailed analysis and a more precise calculation of the intima-media thickness (RFQIMT), the velocity of the pulse wave (VPW) and the degree of endothelial dysfunction at the level of the brachial artery by the FMD method. The abnormal values of which have recently been considered to be evidence of accelerated pathological aging, allows us to have a general overview of damage incurred by the arterial wall and of the state of endothelial function. These patients' assessment should of course be complemented by paraclinical examinations of atheromatosis and its complications. One may even conclude that the removal/regression of the persistent biological 
inflammation, which is itself the cause of the atherosclerotic progression and endothelial dysfunction. These chronic inflammatory diseases, and even more so chronic inflammatory arthritis and connective diseases, should now be considered as new cardiovascular risk factors.

\section{References}

[1] Castellon X, Bogdanova V (2013). Screening for subclinical atherosclerosis by noninvasive methods in asymptomatic patients with risk factors. Clin Interv Aging, 8: 573-580

[2] Xavier Castellon, V.Bogdanova (2011).Study of arterial rigidity QAS: Evaluation by radio frequency of the quality of carotide rigidity on level in real-time, in patients with cardiovascular risk factors. Arch Cardiovasc Dis, 3: 85-86

[3] Lopez LR, Simpson DF, Hurley BL, Matsuura E (2005). OxLDL/beta2GPI complexes and autoantibodies in patients with systemic lupus erythematosus, systemic sclerosis, and antiphospholipid syndrome: pathogenic implications for vascular involvement. Ann N Y Acad Sci, 1051: 313-322

[4] Charniot JC CC, Chemouni F, Bogdanova V (2010). Oxidative stress implications in cardiogenic shock with ischemic or idiopathic severe left ventricular dysfunction: role of etiologies of cardio- myopathies. Archives of Cardiovascular Diseases Supplements, 2

[5] Charniot JC, Vignat N, Albertini JP, Bogdanova V, Zerhouni K, Monsuez JJ, et al. (2008). Oxidative stress in patients with acute heart failure. Rejuvenation Res, 11: 393-398

[6] Sengupta PP, Pedrizzetti G, Kilner PJ, Kheradvar A, Ebbers T, Tonti G, et al. (2012). Emerging trends in CV flow visualization. JACC Cardiovasc Imaging, 5: 305316

[7] X .Castellon (2006). Methods of assessing endothelial function, vasodilation of interest by the intermediate flow. Cardiology Practice, 8: 10-12

[8] Gimbrone MA, Jr. (1995). Vascular endothelium: an integrator of pathophysiologic stimuli in atherosclerosis. Am J Cardiol, 75: 67B-70B

[9] Wang SS, Schadt EE, Wang H, Wang X, Ingram-Drake L, Shi W, et al. (2007). Identification of pathways for atherosclerosis in mice: integration of quantitative trait locus analysis and global gene expression data. Circ Res, 101: e11-30

[10] Blankenberg S, Barbaux S, Tiret L (2003). Adhesion molecules and atherosclerosis. Atherosclerosis, 170: 191-203

[11] Reiner Z, Tedeschi-Reiner E (2001). [New information on the pathophysiology of atherosclerosis]. Lijec Vjesn, 123: $26-31$

[12] Puddu P, Cravero E, Puddu GM, Muscari A (2005). Genes and atherosclerosis: at the origin of the predisposition. Int J Clin Pract, 59: 462-472

[13] Berliner JA, Navab M, Fogelman AM, Frank JS, Demer LL, Edwards PA, et al. (1995). Atherosclerosis: basic mechanisms. Oxidation, inflammation, and genetics. Circulation, 91: 2488-2496

[14] Falk E (2006). Pathogenesis of atherosclerosis. J Am Coll Cardiol, 47: C7-12

[15] Cyrus T, Witztum JL, Rader DJ, Tangirala R, Fazio S, Linton MF, et al. (1999). Disruption of the 12/15lipoxygenase gene diminishes atherosclerosis in apo Edeficient mice. J Clin Invest, 103: 1597-1604

[16] Kullo IJ, Gau GT, Tajik AJ (2000). Novel risk factors for atherosclerosis. Mayo Clin Proc, 75: 369-380

[17] Ruiz-Ortega M, Lorenzo O, Ruperez M, Esteban V, Suzuki Y, Mezzano S, et al. (2001). Role of the reninangiotensin system in vascular diseases: expanding the field. Hypertension, 38: 1382-1387

[18] Duprez DA (2006). Role of the renin-angiotensinaldosterone system in vascular remodeling and inflammation: a clinical review. J Hypertens, 24: 983991

[19] Schiffrin EL, Touyz RM (2004). From bedside to bench to bedside: role of renin-angiotensin-aldosterone system in remodeling of resistance arteries in hypertension. Am J Physiol Heart Circ Physiol, 287: H435-446

[20] Touyz RM (2005). Intracellular mechanisms involved in vascular remodelling of resistance arteries in hypertension: role of angiotensin II. Exp Physiol, 90: 449-455

[21] Lusis AJ, Mar R, Pajukanta P (2004). Genetics of atherosclerosis. Annu Rev Genomics Hum Genet, 5: 189-218

[22] Conlan MG, Folsom AR, Finch A, Davis CE, Sorlie P, Marcucci G, et al. (1993). Associations of factor VIII and von Willebrand factor with age, race, sex, and risk factors for atherosclerosis. The Atherosclerosis Risk in Communities (ARIC) Study. Thromb Haemost, 70: 380385

[23] Mannucci PM (1998). von Willebrand factor: a marker of endothelial damage? Arterioscler Thromb Vasc Biol, 18: $1359-1362$

[24] Beaudeux J-L, Delattre J, P.Therond, BonnefontRousselot D, Legrand A, Peyne J (2006). Le stress oxydant, composante physiopathologique de l'athérosclérose. Immunoanalyse \& Biologie Spécialisée, 21: $144-150$

[25] Towfighi A, Markovic D, Ovbiagele B (2012). Utility of Framingham Coronary Heart Disease Risk Score for predicting cardiac risk after stroke. Stroke, 43: 29422947

[26] Brindle P, Emberson J, Lampe F, Walker M, Whincup P, Fahey T, et al. (2003). Predictive accuracy of the Framingham coronary risk score in British men: prospective cohort study. BMJ, 327: 1267

[27] Lange RA (2013). Can you predict what happens when EuroSCORE weds biomarker? J Am Coll Cardiol, 61: 682-684

[28] Li JJ, Fang CH (2004). C-reactive protein is not only an inflammatory marker but also a direct cause of cardiovascular diseases. Med Hypotheses, 62: 499-506

[29] Rutter MK, Meigs JB, Sullivan LM, D'Agostino RB, Sr., Wilson PW (2004). C-reactive protein, the metabolic 
syndrome, and prediction of cardiovascular events in the Framingham Offspring Study. Circulation, 110: 380-385

[30] Shoenfeld Y, Gerli R, Doria A, Matsuura E, Cerinic MM, Ronda N, et al. (2005). Accelerated atherosclerosis in autoimmune rheumatic diseases. Circulation, 112: 33373347

[31] Doria A, Sarzi-Puttini P, Shoenfeld Y (2006). 2nd conference on heart, rheumatism and autoimmunity, Pescara, Italy, May 19-20, 2005. Autoimmun Rev, 5: 5563

[32] Belizna CC, Richard V, Primard E, Kerleau JM, Cailleux N, Louvel JP, et al. (2008). Early atheroma in primary and secondary antiphospholipid syndrome: an intrinsic finding. Semin Arthritis Rheum, 37: 373-380

[33] He M, Liang X, He L, Wen W, Zhao S, Wen L, et al. (2013). Endothelial dysfunction in rheumatoid arthritis: the role of monocyte chemotactic protein-1-induced protein. Arterioscler Thromb Vasc Biol, 33: 1384-1391

[34] Rey S, Semenza GL (2010). Hypoxia-inducible factor-1dependent mechanisms of vascularization and vascular remodelling. Cardiovasc Res, 86: 236-242

[35] Hulten LM, Levin M (2009). The role of hypoxia in atherosclerosis. Curr Opin Lipidol, 20: 409-414

[36] Drager LF, Bortolotto LA, Lorenzi MC, Figueiredo AC, Krieger EM, Lorenzi-Filho G (2005). Early signs of atherosclerosis in obstructive sleep apnea. Am J Respir Crit Care Med, 172: 613-618

[37] De Vriese AS, Verbeuren TJ, Van de Voorde J, Lameire NH, Vanhoutte PM (2000). Endothelial dysfunction in diabetes. Br J Pharmacol, 130: 963-974

[38] Calles-Escandon J, Cipolla M (2001). Diabetes and endothelial dysfunction: a clinical perspective. Endocr Rev, 22: 36-52

[39] Hink U, Li H, Mollnau H, Oelze M, Matheis E, Hartmann M, et al. (2001). Mechanisms underlying endothelial dysfunction in diabetes mellitus. Circ Res, 88: E14-22

[40] Di Carli MF, Janisse J, Grunberger G, Ager J (2003). Role of chronic hyperglycemia in the pathogenesis of coronary microvascular dysfunction in diabetes. J Am Coll Cardiol, 41: 1387-1393

[41] Festa A, D'Agostino R, Jr., Howard G, Mykkanen L, Tracy RP, Haffner SM (2000). Chronic subclinical inflammation as part of the insulin resistance syndrome: the Insulin Resistance Atherosclerosis Study (IRAS). Circulation, 102: 42-47

[42] Bonora E, Kiechl S, Willeit J, Oberhollenzer F, Egger G, Bonadonna RC, et al. (2003). Carotid atherosclerosis and coronary heart disease in the metabolic syndrome: prospective data from the Bruneck study. Diabetes Care, 26: $1251-1257$

[43] Olechnowicz-Tietz S, Gluba A, Paradowska A, Banach M, Rysz J (2013). The risk of atherosclerosis in patients with chronic kidney disease. Int Urol Nephrol, 45: 16051612

[44] Koike M, Nitta K (2011). [Chronic kidney disease and atherosclerosis]. Nihon Rinsho, 69: 144-150

[45] Doria A, Shoenfeld Y, Wu R, Gambari PF, Puato M, Ghirardello A, et al. (2003). Risk factors for subclinical atherosclerosis in a prospective cohort of patients with systemic lupus erythematosus. Ann Rheum Dis, 62: 1071-1077

[46] Selzer F, Sutton-Tyrrell K, Fitzgerald SG, Pratt JE, Tracy RP, Kuller LH, et al. (2004). Comparison of risk factors for vascular disease in the carotid artery and aorta in women with systemic lupus erythematosus. Arthritis Rheum, 50: 151-159

[47] Thompson T, Sutton-Tyrrell K, Wildman RP, Kao A, Fitzgerald SG, Shook B, et al. (2008). Progression of carotid intima-media thickness and plaque in women with systemic lupus erythematosus. Arthritis Rheum, 58: 835-842

[48] Nicholls SJ, Tuzcu EM, Crowe T, Sipahi I, Schoenhagen P, Kapadia S, et al. (2006). Relationship between cardiovascular risk factors and atherosclerotic disease burden measured by intravascular ultrasound. J Am Coll Cardiol, 47: 1967-1975

[49] Cunningham KS, Gotlieb AI (2005). The role of shear stress in the pathogenesis of atherosclerosis. Lab Invest, 85: $9-23$

[50] Toth PP (2008). Subclinical atherosclerosis: what it is, what it means and what we can do about it. Int $\mathrm{J}$ Clin Pract, 62: 1246-1254

[51] Tsou JK, Liu J, Insana MF (2006). Modeling and phantom studies of ultrasonic wall shear rate measurements using coded pulse excitation. IEEE Trans Ultrason Ferroelectr Freq Control, 53: 724-734

[52] Touboul PJ, Hennerici MG, Meairs S, Adams H, Amarenco P, Desvarieux M, et al. (2004). Mannheim intima-media thickness consensus. Cerebrovasc Dis, 18: 346-349

[53] Huck CJ, Bronas UG, Williamson EB, Draheim CC, Duprez DA, Dengel DR (2007). Noninvasive measurements of arterial stiffness: repeatability and interrelationships with endothelial function and arterial morphology measures. Vasc Health Risk Manag, 3: 343349

[54] Widlansky ME, Gokce N, Keaney JF, Jr., Vita JA (2003). The clinical implications of endothelial dysfunction. J Am Coll Cardiol, 42: 1149-1160

[55] Adji A, O'Rourke MF, Namasivayam M (2011). Arterial stiffness, its assessment, prognostic value, and implications for treatment. Am J Hypertens, 24: 5-17

[56] Singh U, Jialal I (2006). Oxidative stress and atherosclerosis. Pathophysiology, 13: 129-142

[57] Stocker R, Keaney JF, Jr. (2004). Role of oxidative modifications in atherosclerosis. Physiol Rev, 84: 13811478

[58] Bonomini F, Tengattini S, Fabiano A, Bianchi R, Rezzani R (2008). Atherosclerosis and oxidative stress. Histol Histopathol, 23: 381-390

[59] Intengan HD, Schiffrin EL (2001). Vascular remodeling in hypertension: roles of apoptosis, inflammation, and fibrosis. Hypertension, 38: 581-587

[60] Matsuura E, Kobayashi K, Inoue K, Lopez LR, Shoenfeld Y (2005). Oxidized LDL/beta2-glycoprotein I complexes: new aspects in atherosclerosis. Lupus, 14: 736-741

[61] Solomon DH, Karlson EW, Rimm EB, Cannuscio CC, Mandl LA, Manson JE, et al. (2003). Cardiovascular 
morbidity and mortality in women diagnosed with rheumatoid arthritis. Circulation, 107: 1303-1307 